\title{
Spinopelvic parameters and pain in patients with low-grade spondylolisthesis
}

\author{
Hamid Behzadnia', Shahrokh Yousefzadeh-Chabok ${ }^{2,3}$, Babak Alijani', \\ Shabnam Golmohammadi', Zoheir Reyhanian' ${ }^{1}$, Amin Naseri', Sasan Andalib $2,3,4,5,6$ \\ ${ }^{1}$ Department of Neurosurgery, Poursina Hospital, School of Medicine, \\ Guilan University of Medical Sciences, Rasht, Iran \\ ${ }^{2}$ Neuroscience Research Center, Department of Neurosurgery, Poursina Hospital, \\ School of Medicine, Guilan University of Medical Sciences, Rasht, Iran \\ ${ }^{3}$ Department of Nuclear Medicine, Odense University Hospital, Odense, Denmark \\ ${ }^{4}$ Research Unit of Clinical Physiology and Nuclear Medicine, Department of Clinical Research, Faculty of Health \\ Sciences, University of Southern Denmark, Odense, Denmark \\ ${ }^{5}$ BRIDGE-Brain Research-Inter-Disciplinary Guided Excellence, \\ Department of Clinical Research, University of Southern Denmark, Odense, Denmark \\ ${ }^{6}$ Research Unit of Psychiatry, Department of Psychiatry, Psychiatry in the Region of Southern Denmark, \\ University of Southern Denmark, Odense, Denmark
}

\begin{abstract}
Background. Spondylolisthesis is the forward slippage of the upper to the lower vertebrae, which affects spinal cord. Spinal fusion is an important method for the stability of the spine leading to pain and disability reduction in patients with chronic low back pain in spondylolisthesis. The aim of this study was to evaluate postoperative changes in spinopelvic parameters, pain, and disability in low-grade spondylolisthesis patients undergoing posterior lateral fusion (PLF) and posterior lateral interbody fusion (PLIF).

Materials and methods. In the present study, 68 patients who underwent PLF and PLIF due to low-grade spondylolisthesis were recruited. The spinopelvic parameters, visual analogue scale (VAS) score and Oswestry disability index (ODI) before and after surgery were compared.

Results. Pelvic tilt (PT) decreased in both groups after surgery, with more significant decline in PLIF group. Moreover, the mean of PT returned to normal value at both groups. There was also a significant decline in both VAS score and ODI parameters between the two groups. There was a correlation between VAS score and postoperative PT changes. However, the pelvic incidence, lumbar lordosis (PI-LL) and VAS score index did not differ significantly between the two groups after surgery.

Conclusion. Both PLF and PLIF in low-grade spondylolisthesis patients are useful in restoring PT and pelvic stability, but there is no significant difference in the postoperative pain and disability of patients in the two methods.
\end{abstract}

Keywords: spinopelvic parameters, posterior lateral fusion, posterior lumbar interbody fusion, spondylolisthesis

\section{INTRODUCTION}

Spondylolisthesis is the forward slippage of the upper to the lower vertebrae. Spinal fusion is an important method for the stability of the spine leading to pain and disability reduction in patients with chronic low back pain in spondylolisthesis. Its goal is to reduce pain by decreasing segmental motion. In recent studies, the most important investigated factor for short-term clinical improvement of these patients is spinal cord decompression in which short- and long-term clinical outcomes are attributed to sagittal balance and spinopelvic parameter correction (1-5). Sagittal balance preserves muscle activity in the standing position and thus reduces pain (6). Improvement in 
clinical outcome and patient satisfaction after lumbar spinal fusion correlates with postoperative pelvic tilt $(7,8)$ correction of sagittal balance, and increased lumbar lordosis lordosis $(5,6,9)$. Sacral slope and pelvic tilt are also associated with postoperative residual pain $(6,10)$.

Among different spinopelvic parameters, pelvic tilt (PT) has the highest association with quality of life related to health (HRQOL) and pain (7). Posterior lumbar fusion (PLF) and posterior lateral interbody fusion (PLIF) are common methods in the treatment of lumbar spondylolisthesis.

A considerable amount of literature has compared radiological and clinical outcomes in these two methods. In these studies, biomechanical complications such as adjacent level degeneration in lateral posterior fusion surgery and local complications such as surgical wound infections and osteomyelitis have been reported, particularly in the posterior lateral interbody fusion method $(8,11-$ 13). In the previous studies, the maximum lumbar lordosis correction was provided by the posterior lateral interbody fusion $(1,14)$. Although satisfactory clinical outcomes have been reported in a variety of surgical techniques, the preferred method of fusion is still controversial. Numerous studies have failed to reveal any significant differences between fusion techniques.

The aim of this study was to evaluate changes in spinopelvic parameters in PLF and PLIF and their correlation to clinical outcomes assessed by VAS score and Oswestry disability index (ODI).

\section{MATERIALS AND METHODS}

In this study, sixty-eight subjects undergoing lumbosacral fusion surgery for low-grade spondylolisthesis at Poursina Hospital were studied. Patients with a history of lumbar fusion, previous pelvic surgery and lower limb length difference of greater than $2 \mathrm{~cm}$ were excluded. All the patients had been assessed by plain x-ray prior to surgery, as well as follow-ups 3 and 12 months after surgery. The assessment of sagittal balance parameters was done by the Duval-Beaupere's and Cobb's criteria. The evaluation of spinopelvic parameters was performed by a lumbar sacral plain x-ray from T10 to Sacrum in standing position. Arms were held at 45 degrees of flexion and the hip and knee were kept in full extension. The reference point was defined as the head of femur and in the graphs that two femurs were not matched, their interfaces were used instead. Pain was assessed by using visual analogue scale (VAS). The ODI was also assessed. Variables were compared using ANOVA and independent t-tests. Logistic regression analysis was used to investigate the effect of confounding factors. The study was approved by ethics committee of Guilan University of Medical Sciences.

\section{RESULTS}

Sixty-eight patients were enrolled in the study according to relevant inclusion and exclusion criteria. Females and males constituted $80.6 \%$ and $19.4 \%$ of patients, respectively. Twenty-eight percent of the patients had diabetes mellitus. Preoperative mode of spondylolisthesis grade was 2 . Sixty-two percent of the patients were treated by the PLIF and thirty-eight percent by the PLF. Diabetes differed significantly in the PLIF and PLF groups before the surgery $(\mathrm{P}=0.03)$. Gender distribution and spondylolisthesis dispersion in the two groups did not differ significantly $(\mathrm{P}=0.04)$.

The mean follow-up duration for the patients in the PLIF surgery group was 13.6 months, and 14.1 months for the PLF group. The mean age of the patients in the PLF group was 53.7 \pm years and $52.6 \pm$ years in the PLIF group.

It was found that among spinopelvic, VAS, and ODI, only PT and VAS score changes were significantly different in the PLIF and PLF treatment groups (Tables 1 and 2). Specifically, the mean of these variables within the PLF group was significantly high, compared to the PLIF group (Table 3) and both groups reached normal Pelvic incidence Lumbar lordosis parameter, which shows pelvic balance.

TABLE 1. Spinopelvic parameters in PLF and PLIF groups

\begin{tabular}{|c|c|c|}
\hline Spinopelvic parameters & PLF & PLIF \\
\hline Pre-opera & $57.3 \pm 4.2$ & $56.9 \pm 3.2$ \\
\hline $\begin{array}{l}\text { Pelvic incidence } 3 \text { months a er } \\
\text { surgery }\end{array}$ & $54.4 \pm 3.95$ & $52.2 \pm 4.3$ \\
\hline $\begin{array}{l}\text { Pelvic incidence } 12 \text { months a er } \\
\text { surgery }\end{array}$ & $56.3 \pm 4.24$ & $52.7 \pm 2.8$ \\
\hline Pre-opera & $21.6 \pm 5.2$ & $22 \pm 4.1$ \\
\hline ths a er surgery & $13.33 \pm 4.2$ & $12.4 \pm 2.2$ \\
\hline ths a? ?er surgery & $10.35 \pm 4.24$ & $11.5 \pm 2.8$ \\
\hline Pre-opera & $\beta 8.7 \pm 3.2$ & $34.31 \pm 2.4$ \\
\hline
\end{tabular}




\begin{tabular}{|c|c|c|}
\hline Spinopelvic parameters & PLF & PLIF \\
\hline Sacral slope 3 months a er surgery & $40.12 \pm 3.7$ & $34.84 \pm 3.6$ \\
\hline $\begin{array}{l}\text { Sacral slope } 12 \text { months a er } \\
\text { surgery }\end{array}$ & $40 \pm 4$ & $41 \pm 2.5$ \\
\hline Pre-opera & $42.6 \pm 2.5$ & $37.42 \pm 1.6$ \\
\hline $\begin{array}{l}\text { Lumbar lordosis } 12 \text { months a er } \\
\text { surgery }\end{array}$ & $43.2 \pm 2.8$ & $40.61 \pm 2.3$ \\
\hline Pre-opera & $13.66 \pm 1.1$ & $12.65 \pm 1.7$ \\
\hline Post-opera & $12.09 \pm 1.7$ & $10.23 \pm 1.5$ \\
\hline
\end{tabular}

TABLE 2. Pain and disability parameters in PLF and PLIF groups

\begin{tabular}{|c|c|c|}
\hline Pain and disability parameters & PLF & PLIF \\
\hline Pre-opera & $8.87 \pm 0.8$ & $8.7 \pm 1.23$ \\
\hline VAS score 12 months a er surgery & $3.31 \pm 1.5$ & $2.77 \pm 1.28$ \\
\hline Pre-opera & $3.86 \pm 0.74$ & $3.96 \pm 0.85$ \\
\hline ODI 12 months a er surgery & $1.6 \pm 0.63$ & $1.6 \pm 0.83$ \\
\hline
\end{tabular}

TABLE 3. The before-after surgery (Year 1) changes of in spinopelvic parameters, pain, and disability in PLF and PLIF groups

\begin{tabular}{|l|c|c|c|c|}
\hline \multirow{2}{*}{ es } & Group & Mean & $\begin{array}{c}\text { Standard } \\
\text { deviation }\end{array}$ & $\begin{array}{c}\text { Standard } \\
\text { error of } \\
\text { mean }\end{array}$ \\
\hline \multirow{2}{*}{ P } & PLF & 4.46 & 11.6 & 0.76 \\
\cline { 2 - 5 } & PLIF & 0.78 & 11.3 & 0.52 \\
\hline \multirow{2}{*}{$\begin{array}{l}\text { Sacral slope } \\
\text { changes }\end{array}$} & PLF & -1.28 & 7.7 & 0.52 \\
\cline { 2 - 5 } $\begin{array}{l}\text { Lumbar lordosis } \\
\text { changes }\end{array}$ & PLIF & -1.14 & 8.7 & 0.41 \\
\hline \multirow{2}{*}{ VAS score changes } & 1.92 & 10. & 0.61 \\
\cline { 2 - 5 } & PLIF & 1.91 & 10. & 0.47 \\
\cline { 2 - 5 } & PLF & 5.76 & 2.649 & 0.14 \\
\hline \multirow{2}{*}{ ODI changes } & PLF & 6.17 & 1.73 & 0.07 \\
\cline { 2 - 5 } & PLIF & 2.14 & 0.98 & 0.06 \\
\hline
\end{tabular}

\section{DISCUSSION}

In this study, final results of PT were not associated with method of surgery significantly but the changes within a year was more prominent in PLIF group. Our findings corroborated with those of Feng et al. in which in a nonrandomized controlled prospective study on patients with low-grade L5-S1 isthmic spondylolisthesis to evaluate the effect of PLF and PLIF on spinopelvic sagittal balance (1). The authors found that either PLF or PLIF would change dramatically in spinopelvic and deformity parameters. The decline of PT may have a crucial role in the short-term surgical outcome (1). PLIF by increasing the lumbar lordosis produces a more reasonable sagittal alignment in isthmic spondylolisthesis (1).

Kim et al. studied if lumbar sagittal balance influences clinical outcomes subsequent to PLIF and demonstrated that improving PT after fusion results in good clinical outcomes in degenerative spondylolisthesis (2).

Lazennec et al. conducted a radiological analysis of posture before and after lumbosacral fusion for the assessment of the impact of spinal alignment on the post-surgical pain (10). Moreover, at last follow-up, PT was correlated with post-fusion pain and was approximately twice as high as the normal value. ST in the standing position was also correlated with post-fusion pain showing that the vertical sacrum was more prevalent in the cases with post-fusion pain (10). Nevertheless, by using logistic regression, only ST remained prognostic for residual pain (10). The decline was positively correlated with a clinical outcome $(2,10)$, but negatively with post-surgical pain, which was in agreement with our findings.

In this study, post-surgical sacral slope increased non-significantly in both surgical groups; Changes in sacral slope were significantly correlated with those in PI, lumbar lordosis, and PT. These results corroborated the findings of Feng et al. as mentioned above (1). Lazennec et al. illustrated that a smaller ST was associated with post-surgical residual pain (10).

We found that post-surgical lumbar lordosis decreased non-significantly in both groups. Moreover, findings of Feng et al. (1) revealed that lordosis changes were not significant; albeit the study of Musulman et al. (14) showed significant increase in lumbar lordosis in the PLIF group.

In this study, pelvic incidence- lumbar lordosis (PI-LL), which is an index of pelvic stability were 10.2 and 12 degrees in the PLIF and the PLF groups, respectively. Although, in both groups, it returned to normal values, the difference was not significant.

According to the results of previous research, the formula $L L=-(P I+10$ degrees $)$ is an effective method for surgical planning of lordosis and favorable curvature (15) curvature and the index is a predisposing factor in adjacent segmental disease (16) and postoperative sacroiliac joint pain (17).

In this study, the postsurgical ODI decreased in both groups and the difference was non-significant. In the systematic review carried out by Liu et al. (13), the clinical satisfaction and fusion rates in PLIF were higher than PLF. Farrokhi et al. (18) performed a prospective study on ischemic spon- 
dylolisthesis followed by PLIF and PLF for a year and showed that clinical outcomes and pain reduction in the PLF group was significantly better than the PLIF group, despite lower fusion.

Ekman et al. (19) prospectively studied the effect of PLIF and PLF in ischemic spondylolisthesis for 2 years and found that these surgical methods did not differ in patients' clinical outcomes. Elsewhere, a prospective investigation on PLIF and PLF for 4 years fail to show any significant difference in the clinical improvement of subjects based upon ODI and VAS criteria (20). Audat et al. (21) compared the clinical and radiological findings of fusion surgery in PLIF, PLF, and TLIF in the treatment of degenerative spinal spondylolisthesis and demonstrated that clinical improvement of the patients in the three groups did not differ significantly.

In this study, VAS score after surgery decreased in both groups to 6.1 and 5.7 in PLIF in the PLF groups, respectively, and the surgical change (post-operative VAS score minus pre-operative VAS score) was more pronounced in the PLIF group. VAS score changes were correlated with PT and ODI changes. Farrokhi et al., compared clinical outcomes of PLF with PLIF with posterior fusion in isthmic spondylolisthesis, the clinical outcome and ODI of patients in the PLF were superior to the PLIF. There was no difference between the change in spondylolisthesis grade and the radicular pain (18). In addition, no difference between the ODI and VAS of patients in PLF and PLIF $(19,21,22)$. Alijani et al. (23) performed a prospective observational study on low-grade degenerative and isthmic spondylolisthesis comparing PLF and PLIF methods on post-surgical disability using ODI and found a significant declining trend in either groups and both surgical fusion techniques efficiently lessened the disability of patients with spondylolisthesis, notwithstanding a nonsignificant difference.

\section{CONCLUSION}

In sum, PLIF can more effectively correct pelvic tilt; despite the fact that both PLIF and PLF achieved a balanced pelvic index. We recommend as a further investigation considering differentiation of postoperative pain based on sitting, standing, walking postures, and type of axial or radicular pain.

\section{Acknowledgement}

We thank Guilan University of Medical Sciences for its support.

Conflict of interest: none declared Financial support: none declared

\section{REFERENCES}

1. Feng Y, Chen L, Gu Y, Zhang ZM, Yang HL, Tang TS. Restoration of the spinopelvic sagittal balance in isthmic spondylolisthesis: posterior lumbar interbody fusion may be better than posterolateral fusion. Spine J. 2015;15(7):1527-35.

2. Kim MK, Lee S-H, Kim E-S, Eoh W, Chung S-S, Lee C-S. The impact of sagittal balance on clinical results after posterior interbody fusion for patients with degenerative spondylolisthesis: A pilot study. BMC Musculoskeletal Disorders. 2011;12:69.

3. Lee CS, Hwang CJ, Lee DH, Kim YT, Lee HS. Fusion rates of instrumented lumbar spinal arthrodesis according to surgical approach: a systematic review of randomized trials. Clinics in Orthopedic Surgery. 2011;3(1):39-47.

4. Videbaek TS, Bunger CE, Henriksen M, Neils E, Christensen FB. Sagittal spinal balance after lumbar spinal fusion: the impact of anterior column support results from a randomized clinical trial with an eight- to thirteen-year radiographic follow-up. Spine. 2011;36(3):183-91.

5. Elgafy $H$, Bransford R, Semaan H, Wagner T. Clinical and radiographic evaluation of sagittal imbalance: a new radiographic assessment. American Journal of Orthopedics (Belle Mead, NJ). 2011;40(3):E30-4

6. Bourghli A, Aunoble S, Reebye O, Le Huec JC. Correlation of clinical outcome and spinopelvic sagittal alignment after surgical treatment of low-grade isthmic spondylolisthesis. Eur Spine J. 2011;20 Suppl 5:663-8.

7. Kim MK, Lee SH, Kim ES, Eoh W, Chung SS, Lee CS. The impact of sagittal balance on clinical results after posterior interbody fusion for patients with degenerative spondylolisthesis: a pilot study. BMC Musculoskeletal Disorders. 2011;12:69.

8. Li L, Sui H, Yu X, Jiang B. [Correlation of clinical outcome and spinopelvic sagittal alignment after surgical posterior intervertebral fusion combined with pedicle screw fixation for low-grade isthmic lumbar spondylolisthesis]. Chinese Journal of Reparative and Reconstructive Surgery. 2013;27(11):1338-44.

9. Berven SH, Deviren V, Smith JA, Hu SH, Bradford DS. Management of fixed sagittal plane deformity: outcome of combined anterior and posterior surgery. Spine. 2003;28(15):1710-6.

10. Lazennec JY, Ramare S, Arafati N, Laudet CG, Gorin M, Roger B, et al. Sagittal alignment in lumbosacral fusion: relations between radiological parameters and pain. Eur Spine J. 2000;9(1):47-55.

11. Dantas FL, Prandini MN, Ferreira MA. Comparison between posterior lumbar fusion with pedicle screws and posterior lumbar interbody fusion with pedicle screws in adult spondylolisthesis. Arquivos de neuro-psiquiatria. 2007;65(3b):764-70.

12. Ahn DK, Park HS, Choi DJ, Kim TW, Chun TH, Yang JH, et al. The difference of surgical site infection according to the methods of lumbar fusion surgery. Journal of Spinal Disorders \& Techniques. 2012;25(8):E230-4

13. Liu X, Wang Y, Qiu G, Weng X, Yu B. A systematic review with meta-analysis of posterior interbody fusion versus posterolateral fusion in lumbar spondylolisthesis. Eur Spine J. 2014;23(1):43-56. 
14. Musluman AM, Yilmaz A, Cansever T, Cavusoglu H, Colak I, Genc $\mathrm{HA}$, et al. Posterior lumbar interbody fusion versus posterolateral fusion with instrumentation in the treatment of low-grade isthmic spondylolisthesis: midterm clinical outcomes. J Neurosurg Spine. 2011;14(4):488-96.

15. Berjano P, Langella F, Ismael MF, Damilano M, Scopetta S, Lamartina C. Successful correction of sagittal imbalance can be calculated on the basis of pelvic incidence and age. Eur Spine $\mathrm{J}$. 2014;23 Suppl 6:587-96.

16. Matsumoto T, Okuda S, Maeno T, Yamashita T, Yamasaki R, Sugiura $\mathrm{T}$, et al. Spinopelvic sagittal imbalance as a risk factor for adjacentsegment disease after single-segment posterior lumbar interbody fusion. J Neurosurg Spine. 2017;26(4):435-40.

17. Shin MH, Ryu KS, Hur JW, Kim JS, Park CK. Comparative study of lumbopelvic sagittal alignment between patients with and without sacroiliac joint pain after lumbar interbody fusion. Spine (Phila Pa 1976). 2013;38(21):E1334-41.

18. Farrokhi MR, Rahmanian A, Masoudi MS. Posterolateral versus posterior interbody fusion in isthmic spondylolisthesis. Journal of Neurotrauma. 2012;29(8):1567-73.
19. Ekman P, Moller H, Tullberg T, Neumann P, Hedlund R. Posterior lumbar interbody fusion versus posterolateral fusion in adult isthmic spondylolisthesis. Spine. 2007;32(20):2178-83.

20. Cheng $L$, Nie L, Zhang L. Posterior lumbar interbody fusion versus posterolateral fusion in spondylolisthesis: a prospective controlled study in the Han nationality. Int Orthop. 2009;33(4):1043-7.

21. Audat Z, Moutasem O, Yousef K, Mohammad B. Comparison of clinical and radiological results of posterolateral fusion, posterior lumbar interbody fusion and transforaminal lumbar interbody fusion techniques in the treatment of degenerative lumbar spine. Singapore Medical Journal. 2012;53(3):183-7.

22. Ye YP, Xu H, Chen D. Comparison between posterior lumbar interbody fusion and posterolateral fusion with transpedicular screw fixation for isthmic spondylolithesis: a meta-analysis. Archives of Orthopaedic and Trauma Surgery. 2013;133(12):1649-55.

23. Alijani B, Emamhadi M, Behzadnia H, Aramnia A, Chabok SY, Ramtinfar S, et al. Posterior lumbar interbody fusion and posterolateral fusion: Analogous procedures in decreasing the index of disability in patients with spondylolisthesis. Asian Journal of Neurosurgery. 2015;10(1):51. 